\title{
Mental disorders in the Eastern Mediterranean Region
}

\author{
Atif Rahman ${ }^{1}$
}

Received: 18 May 2017/ Accepted: 22 May 2017/Published online: 3 August 2017

(c) Swiss School of Public Health (SSPH+) 2017

Keywords Mental health · Eastern Mediterranean region . Global mental health $\cdot$ Burden from mental disorder

The key finding from Charara and Mokdad (2017) are stark but not surprising: Mental and substance use disorders are the leading cause of non-fatal burden in the Eastern Mediterranean Region (EMR). The rate of mental disorder burden is higher than the global average for almost all EMR countries. There is a clear trend of increasing burden of mental disorders in the EMR from 1990 to 2015, and this increase is particularly striking in the richer countries of the region. Females contribute more to the burden of mental disorders in the EMR compared to males. Treatable conditions such as depression and anxiety account for most of the burden.

What is more surprising is that despite this upward trend over the last two decades, there has been very little investment in mental health services in the region. The World Health Organization's mental health Atlas exercise conducted in 2014 found that even though a substantial number of EMR countries have recently published mental health policies, more than half of these were not fully compliant with international human rights instruments and none were fully implemented. Several countries had

This editorial is part of the supplement "The state of health in the Eastern Mediterranean Region, 1990-2015."

Atif Rahman

atif.rahman@liverpool.ac.uk;

https://www.liverpool.ac.uk/

1 Department of Psychological Sciences, Institute of Psychology, Health and Society, University of Liverpool, Block B, Waterhouse Building, 1-5 Dover Street, Liverpool L69 3BX, UK outdated legislation about mental health, much of which did not conform with international human rights instruments. The involvement of associations of service users and their carers in the formulation and implementation of mental health policies, laws and services was markedly underdeveloped in the region. The poorest countries in the region have seen a fall in the available workforce, probably as a consequence of conflict, political instability and displacement. Despite an increase in the number of out-patients, there was a reduction in the number of out-patient facilities compared to 2011 , and the availability of mental health day-care or day-treatment facilities remained ten times lower than the global average across the region. Most countries had limited research evidence with which to inform service planning or to monitor implementation.

However, there are some reasons to be optimistic. In response to the comprehensive Mental health action plan 2013-2020 adopted by the World Health Assembly in May 2013 (World Health Organization 2013), the WHO EMRO have developed a regional framework identifying high impact, cost-effective, affordable, feasible strategic actions supported by a set of indicators to monitor the implementation of the plan (Alwan and Saeed, 2015; Gater et al. 2015). The framework was adopted by member countries at the Regional Meeting of the Eastern Mediterranean Region in October 2015. Consequently, many countries have begun to provide basic mental health care training to their primary care staff to fill the workforce gap. Mental health promotion and prevention was also identified as an important strategy and a school mental health programme has been developed for phased implementation in the region. While the regional framework is an important step in the process of implementing the comprehensive mental health action plan, its implementation will have to be carefully monitored through future editions of the mental 
health atlas and other evaluations during the next 5 years, and the framework will need to be reviewed and revised in the light of progress.

A neglected area in the region is research that can inform the implementation of evidence-based interventions to reduce the treatment gap for mental disorders and promote mental health in the region. Many countries in the region are affected by conflict and political unrest. Provision of evidence-based basic mental health care at a public health level in such circumstances presents unique challenges. A research programme conducted in Peshawar, Pakistan, in an area affected by armed conflict, aimed to evaluate the effectiveness of a WHO brief transdiagnostic intervention that could be delivered by non-specialists working in primary care centres under supervision of specialists (Rahman et al. 2016). In a randomized clinical trial, 346 adults impaired by psychological distress were randomized to the intervention or enhanced usual care. After 6 weekly sessions of treatment, the intervention group had significantly lower anxiety and depression scores and lower rates of depressive disorder compared with those in the enhanced usual care group. The research showed that this non-specialist administered intervention may be a practical approach for treating adults with psychological distress in conflict-affected areas. This manualized intervention is now being disseminated globally by the WHO (http://www.who.int/mental_health/emergencies/pro blem_management_plus/en/) and is an example of an EMRled research that is valuable in informing new approaches to the unprecedented global challenges in mental health. Another initiative is the NIMH-funded SHINE project (School health Implementation Research Network in the Eastern Mediterranean Region) that works in partnership with the WHO EMRO to build capacity in school mental health research in the region (see https://www.nimh.nih.gov/ about/organization/gmh/scaleuphubs/school-health-imple mentation-network-eastern-mediterranean-region-shine. shtml). However, much more is needed to build further research capacity in the region to allow evidence-based interventions to be developed and scaled-up. It is high-time that the rich countries of the region invested in this critical area.

In the last decades, the EMR has had more than its share of natural and man-made disasters. The region has some of the worst inequalities of wealth distribution. These factors have a long-lasting impact on mental health. Unless concerted efforts are made to address this urgently today, the region will continue to see an increase in the burden of mental disorder in decades to come.

\section{References}

Alwan A, Saeed K (2015) A new agenda for mental health in the Eastern Mediterranean Region. EMHJ 20(7):459-460

Charara RH, Mokdad A (2017) The burden of mental disorders in the Eastern Mediterranean Region, 1990-2015: findings from the Global Burden of Disease study 2015

Gater R, Saeed K, Rahman A (2015) From plan to framework: the process for developing the regional framework to scale up action on mental health in the Eastern Mediterranean Region. EMHJ 20(7):464-466

Rahman A, Hamdani SU, Awan NA, Bryant RB, Dawson KS, Khan MF, Azeemi MM, Akhtar P, Nazir H, Chiumento C, Sijbrandij M, Wang D, Farooq S, van Ommeren M (2016) Effect of a multicomponent behavioral intervention in adults impaired by psychological distress in a conflict-affected area of Pakistan a randomized clinical trial. JAMA 316(24):2609-2617

World Health Organization (2013) Mental health action plan 2013-2020, Geneva. http://www.who.int/mental_health/publica tions/action_plan/en/. Accessed 16 May 2017 